\title{
Nursing Care of 13 Cases with Acute Retinal Necrosis Associated with HIV/AIDS during Day Surgery
}

\author{
Xiaoqun Fang, Jierong Lin, Yu Zhang, Shiyi Deng, Huiming Xiao, Yu Lian* \\ Department of Fundus Surgery, Zhongshan Ophthalmic Center, Sun Yat-sen University, State Key Laboratory of Ophthalmology, \\ Guangzhou, China \\ Email: ^lianyu@gzzoc.com
}

How to cite this paper: Fang, X.Q., Lin, J.R., Zhang, Y., Deng, S.Y., Xiao, H.M. and Lian, Y. (2021) Nursing Care of 13 Cases with Acute Retinal Necrosis Associated with HIV/AIDS during Day Surgery. Open Journal of Nursing, 11, 591-599. https://doi.org/10.4236/ojn.2021.117050

Received: June 9, 2021

Accepted: July 17, 2021

Published: July 20, 2021

Copyright () 2021 by author(s) and Scientific Research Publishing Inc. This work is licensed under the Creative Commons Attribution International License (CC BY 4.0).

http://creativecommons.org/licenses/by/4.0/

\begin{abstract}
Objective: Summarizing the nursing points from the treatment of the 13 cases of AIDS-related acute retinal necrosis syndrome (ARNS). Methods: Retrospective analysis of clinical nursing process of 13 cases (15 eyes) of AIDS-related ARNS patient treated in our hospital from January to December 2019. Results: The retina of all the patients were flat after operation. Postoperative visual acuity: bilateral visual acuity $<0.05$ in 3 cases, $>0.1$ in 11 eyes, $0.01-0.1$ in 3 eyes, $>0.1$ in 1 eye. No adverse events and nursing safety events occurred. Patients recovered well after operation during the follow-up after discharge. Conclusion: The focus of nursing care for patients with HIV/AIDS combined with acute retinal necrosis during daytime surgery is multifaceted, apart from the daily, perioperative nursing in day-care unit, continuous nursing, psychological counseling, disinfection and isolation should be paid attention to. At the same time, with the help of promotion and application of information education means, the treatment compliance of patients can be improved, which plays an essential role in the advance of the treatment effect.
\end{abstract}

\section{Keywords}

HIV/AIDS, Acute Retinal Necrosis, Day Surgery, Nursing

\section{Introduction}

Acquired immune deficiency syndrome (AIDS) is a serious infectious disease caused by human immunodeficiency virus (HIV) infection, which causes progressive damage to human immune function and is prone to various opportunistic infections [1]. AIDS patients may have a variety of ocular opportunistic 
infections in late stage. Acute retinal necrosis syndrome (ARNS) is one of the most common ocular complications in AIDS patients [2], and it is a kind of viral uveitis. The disease is characterized by acute onset, rapid progression and poor prognosis. It often presents as severe vitreocapsulitis, retinal vasculitis and retinal necrosis. The incidence of advanced dvancedretinal detachment is as high as $75 \%$ [3]. Early antiviral therapy for ARNS can effectively control the progression of the disease. If the progression of the lesion is out of control, prophylactic vitrectomy should be considered even though no significant retinal detachment is observed. Advanced retinal detachment is mainly treated by vitrectomy combined with silicone oil tamponade [4]. But the nursing strategies of these AIDS-related acute retinal necrosis syndromes (ARNS) with advanced retinal detachment are not exactly the same with those without AIDS, considering that their ophthalmic conditions, psychological conditions and physical conditions are all different from the usual type of ARNS with retinal detachment. Author of this article retrospectively analyzed the clinical nursing process of 13 patients with HIV/AIDS complicated with ARNS who were admitted to the Fundus Surgery Department of Zhongshan Ophthalmic Center, Sun Yat-sen University during the day surgery. The particular nursing strategies of these AIDS-related ARNS with advanced retinal detachment are presented in this report.

\section{Data and Methods}

Clinical data: From January to December 2019, 13 patients (15 eyes) (Table 1) with HIV/AIDS-related ARNS were taken into the retrospective analysis. All the patients were treated in the day-care unit of Fundus Surgery Department of Zhongshan Ophthalmic Center, Sun Yat-sen University. All patients were hospitalized for 1 day and were discharged within 24 hours. There were 9 males and 4 females. The average age was 43.07 years from 23 to 66 years old. There were 2 cases with binocular vision $<0.05$, with single eye perception index of 7 eyes, 6 eyes with $0.01-0.1$, and 2 eyes with $0.1-0.3$. There were 2 cases with binocular onset, and 15 eyes had retinal detachment.

\section{Effects}

13 patients (15 eyes) accepted vitreoretinal surgery (Table 2). All 13 patients had retinal flatness after surgical treatments. Visual acuity on the first day after surgery: There were 3 cases with both eyes $<0.05$, with postoperative visual perception index of 11 eyes, 3 eyes with $0.01-0.1$, and 1 eye with $>0.1$. After surgical treatment and nursing by medical staff, were taught the basic knowledge of post-operative body position and, mastered the relevant knowledge of diseases and nursing, thus patients have had a positive attitude to deal with the disease. During the inpatient time, no adverse event or nursing safety incident occurred. After discharge, patients were followed up for 3 to 6 months, and no retinal detachment occurred, 10 of whom were followed up through outpatient service, and 3 of whom were actively treated for systemic diseases in local hospitals. 
Table 1. Individual information $(\mathrm{n}=13)$.

\begin{tabular}{llcc}
\hline \multirow{2}{*}{ Gender } & \multicolumn{1}{c}{ Content } & Cases & Percentage (\%) \\
\cline { 2 - 2 } Age & Male & 11 & $84.62 \%$ \\
& Female & 2 & $15.38 \%$ \\
& $20-30$ & 3 & $23.08 \%$ \\
& $30-40$ & 2 & $15.38 \%$ \\
& $40-50$ & 4 & $30.77 \%$ \\
\multirow{3}{*}{ Education } & $50-60$ & 3 & $23.08 \%$ \\
& Above 60 & 1 & $7.69 \%$ \\
& Primary school or below & 4 & $30.77 \%$ \\
& Junior high & 4 & $30.77 \%$ \\
& Senior high or secondary specialized school & 4 & $30.77 \%$ \\
& Junior college or above & 1 & $7.69 \%$ \\
& unmarried & 3 & $23.08 \%$ \\
& Married & 8 & $61.54 \%$ \\
& divorced & 2 & $15.38 \%$ \\
\hline
\end{tabular}

Table 2. Surgical information $(n=15)$.

\begin{tabular}{|c|c|c|c|c|}
\hline & Content & Added content & Cases & Percentage (\%) \\
\hline \multirow[t]{6}{*}{ Operation } & \multirow{4}{*}{ Vitrectomy (a) } & - & 1 & $6.67 \%$ \\
\hline & & Oil injection & 5 & $40.00 \%$ \\
\hline & & Cataract surgery & 2 & $13.33 \%$ \\
\hline & & Intravitreal injection & 2 & $13.33 \%$ \\
\hline & $\begin{array}{l}\text { Conventinal } \\
\text { scleral } \\
\text { buckling } \\
\text { surger (b) }\end{array}$ & & 2 & $13.33 \%$ \\
\hline & $a+b$ & Oil injection & 3 & $20.00 \%$ \\
\hline \multirow[t]{4}{*}{$\begin{array}{l}\text { Post-operative } \\
\text { position }\end{array}$} & & Face-down & 11 & $73.33 \%$ \\
\hline & & Left or right-sided lying & 2 & $13.33 \%$ \\
\hline & & fowler & 1 & $6.67 \%$ \\
\hline & & Changing ( 2 or more) & 1 & $6.67 \%$ \\
\hline
\end{tabular}

\section{Nursing Strategies}

\subsection{Perioperative Nursing Characteristics in Day-Care Units}

1) Nurse-patient integrated whole nursing model. The primary nurse should follow up the whole perioperative nursing of patients during day surgery, and establish a good communication relationship with patients. Nurses should follow up the standard prevention for medical staff. The medical articles used by patients should be disinfected and the environment should be quarantined. In the 
process of nursing, nurses should pay attention to protecting the personal privacy of AIDS patients, avoid talking about their conditions casually, and choose an independent and quiet environment to explain the condition and treatment to them [5], so as to provide patients with a sense of security in medical treatment as much as possible.

2) Evaluation and measures of personalized nursing. Given the short hospitalization time in day-care units, (inpatient time is 1 day and the patient will be discharged within 24 hours), the primary nurses should focus on the evaluation of patients' visual acuity, psychological state, disease awareness, the educational level of patients and their families and give personalized education in addition to improving the general admission evaluation. The education should focus on HIV and ARNS, vitreoretinal perioperative health education, safety guidance for fall prevention for low vision patients, etc.

3) Basic nursing. As ARNS is an infection disease, resulting in low immune function, patients should be placed in single rooms or independent areas as far as possible to avoid infection. To be well ventilated, carry out disinfection and reduce unnecessary personnel entry and exit of the unit.

4) Postoperative nursing for patients during the daytime. The primary nurses should prepare postoperative day-care units for patients and give personalized postoperative education. a) Safety protection of surgical eyes. To guide patients not to collide or rub the surgical eyes. Do not disassemble or wet the dressing on the day of the surgery to avoid infection. b) Post-operative position. Nurses should inform patients that most of them were filled with fillers after vitrectomy, and therapeutic top pressure nursing should be maintained to achieve the purpose of retinal reattachment. Position guidance can be carried out according to specific fillers and retinal detachment areas. c) Disease observation. Nursing staff should clearly inform patients of possible postoperative complications and treatment plans after the discharge so that patients can understand the key points of postoperative self-observation. d) HIV/AIDS medication guidance. Antiviral therapy is the first-line treatment plan of ARNS. Patients are informed of the importance of long-term and adequate antiviral therapy. They should not reduce or stop the drug by themselves, and the medication can be adjusted according to the eye condition. To guide patients to observe their condition and prevent the occurrence of adverse reactions. Patients should observe any adverse drug reactions such as headache, gastrointestinal reaction and rash. During medication, liver and kidney function and blood routine should be monitored regularly, and they should return to the hospital in time when there are adverse reactions [6].

5) Daytime unit discharge evaluation [7]. After the patient passes the discharge evaluation of day surgery, the nurse should clearly inform him or her the time and place of reexamination, confirm the mastery of postoperative position, and go through discharge formalities.

6) Continuous nursing in day-care units. In the model of day surgery, conti- 
nuous nursing is of great significance to ensure patients' safety, improve their experience, and meet their needs for hospitalization, which should be focused on the change from traditional hospital nursing to home nursing [8] [9]. The key points of continuous nursing for AIDS patients complicated with ARNS are as follows: a) Return visit timing: Two hours after the discharge, nurses should pay a return visit on the day of surgery to find out whether the patients have returned to their residences safely and whether there are discomfort symptoms. 1 week, 1 month, 3 months, 6 months after discharge, patients should return to the hospital for review to follow the doctor's advice. b) To focus on postoperative visual acuity, intraocular pressure and recurrence of retinal detachment. c) Postoperative medication and position compliance. d) Regular observation of the ocular manifestation of HIV carriers is helpful for early detection of complications. It is necessary to regularly understand the patients' eye conditions and popularize the relevant knowledge of AIDS protective measures. e) Nursing for patients with low vision [10]. To inform patients to pay attention to potential safety hazards in daily life, place items in a fixed position, go out with family members or caregivers, so as to prevent accidents such as falling, crush and scald.

\subsection{Application of Video Education in Day-Care Units}

Research shows that providing health education for patients with ophthalmic day surgery in line with the characteristics of day care can improve patients' awareness of surgery related knowledge and perioperative health knowledge [11]. The day-care unit of Fundus Surgery Department has been fully and centrally propagandized in information-based videos, and offered unique Q\&As at different time. 1) Video production: The knowledge of perioperative fundus surgery at different time is shown in videos, which are divided into preoperative and intraoperative, postoperative, postural nursing and discharge education. And patients can watch them by scanning WeChat QR code. 2) Time of promotion and education a) Centralized: Put health education prior to the patient's scheduled surgery period. When a surgery is needed, the appointment nurse distributes a notice of appointment surgery, which has a written explanation and a QR code for perioperative health education of fundus surgery, instructing the patient to scan it to learn about the knowledge of perioperative surgery. Of the 13 patients in this report, $73.33 \%$ patients are told to keep their face down and $6.67 \%$ should change their position regularly. b) Personalized: Promotion and education lie in every link of perioperative period, especially in personalized posture nursing education for postoperative patients that should be given corresponding posture guidance according to vitreoretinal surgery. This is a combination of on-site teaching and information education, to teach patients and their families the key points of postoperative posture on the spot, and at the same time guide them to scan the posture QR code for learning. The primary nurse will answer personally if the patient has any question. 


\subsection{Mental Nursing}

HIV/AIDS patients often suffer from depression, anxiety, fear and other psychological problems [12]. According to reports, the psychological reaction of ARNS patients can be divided into four psychological stages: Ignorance, depression, dejection, acceptance [13]. Psychological nursing should be carried out in stages according to the psychological characteristics of patients with HIV/AIDS complicated with ARNS. a) Depression: Patients have a basic understanding of the disease, lack confidence in the condition and visual prognosis, and may have excessive behavior when they do not cooperate with treatment. Nurses should take the initiative to communicate, strengthen the education and protection of patients, and prevent excessive behavior. b) Dejection: Repeated illness makes the patient indifferent, silent and disheartened in treatment. Using positive body language to give psychological support, nurses can listen to patients' feelings, mobilize their family to support the patient, educate the knowledge of AIDS and ARNS disease for patients' families, to eliminate their fear, and improve patients' treatment compliance. c) Acceptance: The patient is on the mend and gradually transitioned to the acceptance stage, but paid less attention on the disease as his compliance declines. Nurses should strengthen promotion and education of compliance and inform patients of the importance of insisting on treatment and regular reexamination. According to the hospital nursing evaluation, 3 of the 13 surgical patients suffered from depression, 5 were in dejected stage and 5 were in acceptance stage. Familial support is an important part for patients to deal with the disease better, $61.54 \%$ of the 13 patients are married. Their spouses can take better care of the patients, mentally as well as physically. After psychological counseling, 13 patients were able to coordinate the nursing, accept the surgery, strengthen the compliance of postoperative treatment cooperation, and enhance the confidence of treatment.

\subsection{Standard Prevention, Disinfection and Isolation of Day-Care Unit}

The day-care unit is featured with "short, frequent and fast", and patients need a number of diagnoses and treatments before surgery. Thus, in addition to the safety of perioperative patients, medical staff should take preventive measures and disinfection and isolation according to standards.

\subsubsection{Standard Protection for Ophthalmic Medical Staff}

A study has shown that tears of HIV infectors may carry the virus, therefore, ophthalmic medical staff should keep high vigilance against this common extracorporeal secretion when contacting with these patients [14]. Medical staff should strengthen the prevention training of blood-transmitted diseases, and should do the following operations: 1) Using eye-drops/ointment: Avoid eyes contacting with drug nozzle, tears and mucous membrane, and patients do not use the same eye-drops. 2) Eye examinations: Wash hands immediately after 
measuring intraocular pressure and other eye examinations that may contact with tears, mucous membranes or other body fluids of patients contacting patients' eyes, or after contacting different patients. Examination instruments or devices directly contacting with patients' eyes should be disinfected and sterilized before reuse. 3) Invasive nursing manipulation: Disposable gloves are required for intravenous infusion, subconjunctival injection, lacrimal duct flushing and conjunctival sac flushing. The staff must wash hands before and after contacting with patients and wearing gloves. In lacrimal duct washing, given that the patient's body fluid or flushing fluid may contact with the operator's face, gloves, medical masks and protective glasses should be worn [15]. In case of body fluid or flushing fluid, the protection or equipment should be replaced immediately according to the disinfection and isolation system and disinfected. 4) To avoid transmission among patients, the examination and treatment of HIV/AIDS patients should be conducted at the end as far. 5) If nursing staff's hand skin is damaged, their work should be adjusted in time to avoid contacting with blood, body fluids or mucous membrane as much as possible. If contacting is needed, the injured skin must be protected with water-proof bandage before wearing double layer gloves for operation.

\subsubsection{Disinfection and Isolation of Day-Care Unit}

In accordance with the hospital's Standards for Disinfection and Isolation of Blood-Transmitted Infectious Diseases [16], the disinfection and isolation of HIV/AIDS patients in the fundus surgery day-care unit are conducted from three levels: isolation measures, pollutant disposal and terminal disinfection. The diagnosis and treatment items contacted in preoperative and postoperative examinations must take terminal disinfection according to the specifications, with focus on the diagnosis and treatment items for eye examination, including slit lamp, tonometer, three-mirror lens and other instruments contacted with patients.

\section{Conclusion}

ARNS is a potential blinding eye disease, which needs to be paid more attention because of its rapid progress. Despite great progress in the diagnosis and treatment of ARNS, the overall prognosis of ARNS patients with HIV/AIDS is still ineffective due to immune system defects. In recent years, with the rapid development of fundus day surgery, day-care units for the patients are increasing year by year. How to take a systematical treatment and nursing for patients is a common challenge facing nursing staff. Since the day operation ward of fundus, our center has gained a lot of experience in the treatment and nursing of patients with retinal detachment caused by ARNS and HIV/AIDS. The 13 patients included in this paper, the effect of admission education is good, there are no adverse events and nursing safety events in the process of hospitalization, and patients' compliance is good after discharge. Nurses must should take responsibili- 
ty of perioperative nursing, extended nursing, psychological counseling, standard precautions, sterilization and isolation, nursing focus in day surgery, combined with promotion on the Internet, which plays an important role in improving the treatment compliance of ARNS patients and consolidating the treatment effect.

\section{Conflicts of Interest}

The authors declare no conflicts of interest regarding the publication of this paper.

\section{References}

[1] Li, L., Gu, X., Jing, Y.J., et al. (2016) Correlation of CD4+ T Lymphocyte Level with Opportunistic Infections and Viral Load in AIDS Patients Infected with HIV-1. China Journal of Modern Medicine, No. 2, 13-18.

[2] Chronister, C.L., Kaiser, H. and Pagani, J.M. (2011) Progressive Outer Retinal Necrosis in a Patient with Acquired Immune Deficiencysyndrome. Clinical and Experimental Optometry, 94, 389-392. https://doi.org/10.1111/j.1444-0938.2011.00594.x

[3] Takase, H., Okada, A.A., Goto, H., et al. (2015) Development and Validation of New Diagnostic Criteria for Acute Retinal Necrosis. Japanese Journal of Ophthalmology, 59, 14-20. https://doi.org/10.1007/s10384-014-0362-0

[4] Liu, S., Wang, D. and Zhang, X. (2018) The Necessity and Optimal Time for Performing Pars Plana Vitrectomy in Acute Retinal Necrosis Patients. BMC Ophthalmology, 18, Article No. 15. https://doi.org/10.1186/s12886-018-0674-9

[5] Luo, H.M. and Lai, Z.F. (2013) Nursing Care of Patients with AIDS Complicated with Fundus Disease. Huaxi Medicine, 28, 124-125.

[6] Di, Y. and Ye, J.J. (2018) Research Progress on the Diagnosis and Treatment of Acute Retinal Necrosis. Chinese Journal of Ophthalmology, 54, 306-311.

[7] Huang, L.Q., He, Q.G. and Lu, S.F. (2020) Nursing Care of SBL-3 Region Refractive Multifocal Intraocular Lens Implantation during Day Surgery. Contemporary Nurse (SME), 27, 95-97.

[8] Jun, E.Y. and Oh, H. (2017) Patient Needs and Satisfaction with Nursing Care after Day Surgery Based on a Patient-Centered Care Framework. Clinical Nursing Research, 26, 301-317. https://doi.org/10.1177/1054773816633656

[9] Li, Y., Liu, X.J. and Liu, S.X. (2019) Investigation on Quality Evaluation of Transitional Nursing Care for Patients Undergoing Ophthalmic Day Surgery. Chinese Journal of Modern Nursing, 25, 1955-1958.

[10] Lu, S.F., Wu, S.H. and Huang, S.J. (2012) Nursing Care of Patients with Low Vision. Chinese Journal of Practical Nursing, 28, 51-52.

[11] Gu, C.H., Zhu, J.H. and Fu, S.J. (2019) Effect of Diversified Education Model on Health Awareness and Satisfaction of Patients Undergoing Ophthalmic Day Surgery. Chinese Journal of Modern Nursing, 25, 2391-2393.

[12] Dejman, M., Ardakani, H.M. and Malekafzali, B. (2015) Psychological, Social, and Familial Problems of People Living with HIV/AIDS in Iran: A Qualitative Study. International Journal of Preventive Medicine, 6, 126. https://doi.org/10.4103/2008-7802.172540

[13] Li, X.M., Li, X.Y., Sui, Y.W., et al. (2010) Treatment and Nursing of 37 Cases of 
Acute Retinal Necrosis Syndrome. Chinese Journal of Modern Nursing, No. 8, 951-952.

[14] Du, K.F. and Guo, C.G. (2018) AIDS-Associated Fundus Disease. Chinese Medical Journal, 53, 854-857.

[15] Zhang, Q. and Zhang, B. (2016) Standard Preventive Nursing Care of HIV Positive Patients. Shanxi Medical Journal, 45, 1272.

[16] Ouyang, M.L. and Yu, L.N. (2019) Establishment of Nursing Management Standards in Ophthalmology and Preventive Operation Procedure Effectiveness of Reducing Occupational Exposure. Eye Science, 34, 242-249. 